\title{
Dizziness, Vertigo, and Mental Health Comorbidity in Gulf War Veterans
}

DOI: $10.3766 /$ jaaa.17122

\author{
Apollonia Fox* \\ Kristal Riska $\dagger$ \\ Chin-Lin Tseng* \\ Kelly McCarron* \\ Serena Satcher* \\ Omowunmi Osinubi* \\ Drew Helmer*
}

\begin{abstract}
Background: Gulf War Illness (GWI) is a chronic condition involving symptoms across multiple body systems. Previous research has implicated the vestibular system as a potential underlying factor in the symptoms experienced by veterans with GWI, due in part to exposure to potentially ototoxic chemicals and events.
\end{abstract}

Purpose: To characterize the presence of vertigo and dizziness symptoms in a sample of veterans with GWI using validated self-report instruments, accounting for mental health comorbidities.

Research Design: This is a case series, follow-up, prospective interview of clinical veterans; results presented are purely descriptive.

Study Sample: Our sample of 50 veterans was a follow-up to a case series of clinical Gulf War veterans evaluated at the War Related IIIness and Injury Study Center.

Data Collection and Analysis: Veterans participated in a 70-min phone interview where the following questionnaires were administered: Vertigo Symptom Scale (VSS), Patient Health Questionnaire (depression scale), Patient Health Questionnaire (somatization scale), Beck Anxiety Inventory, Posttraumatic Stress Disorder (PTSD) Checklist, Defense and Veterans Brain Injury Center Traumatic Brain Injury Questionnaire, and GWI (Kansas) Questionnaire. We used descriptive (mean/median, standard deviation, interquartile range, and percentage) statistics to describe our sample and illuminate possible relationships between measures.

Results: Our primary finding is a substantial report of vertigo symptoms in our sample, according to the VSS. Ninety percent of participants scored above the VSS threshold (>12), suggesting "severe dizziness." The most commonly endorsed symptom on the VSS was "headache or pressure in the head."

Conclusions: We conclude that there is significant burden of vertigo symptoms in veterans with GWI, suggesting a need for objective tests of vestibular function in this population. Furthermore, the relationship between symptoms of vertigo and dizziness, vestibular function, and PTSD warrants further exploration using objective measures.

Key Words: dizziness, Gulf War Illness, posttraumatic stress disorder, vertigo, veterans

*War Related IIIness and Injury Study Center, New Jersey Health Care System, East Orange, NJ; †Division of Head and Neck Surgery and Communication Sciences, Department of Surgery, Duke University School of Medicine, Durham, NC; $\ddagger$ Rutgers New Jersey Medical School, Newark, NJ

Corresponding author: Apollonia Fox, National Center for Rehabilitative Auditory Research, Portland, OR 97239; Email: apolloniafox@gmail.com

This material is the result of work supported with resources and the use of facilities at the East Orange New Jersey VA medical facility.

The results of this paper have not been previously presented in any form.

The authors declare that the following work was conducted in the absence of any commercial or financial relationships that could be construed as potential conflict of interest.

The views expressed in this article are those of the authors and do not necessarily reflect the position or policy of the Department of Veterans Affairs or the United States government. 


\begin{abstract}
Abbreviations: BAI = Beck Anxiety Inventory; DSM-5 = Diagnostic and Statistical Manual of Mental Disorders, Fifth Edition; GWI = Gulf War Illness; PCL-5 = Posttraumatic Stress Disorder Checklist; PHQ-8 = Personal Health Questionnaire depression scale; PHQ-15 = Personal Health Questionnaire somatization scale; PTSD = posttraumatic stress disorder; $\mathrm{TBI}=$ traumatic brain injury; VA = Veterans Affairs; VSS = Vertigo Symptom Scale; WRIISC = War Related IIIness and Injury Study Center
\end{abstract}

\section{BACKGROUND}

G ulf War Illness (GWI) is a diagnosis of exclusion and is characterized by chronic symptoms across multiple body systems. The Centers for Disease Control and Prevention (CDC) has defined GWI as a history of military service in the Persian Gulf along with a self-report of current symptoms in at least two of the following domains for a duration of more than six months: cognitive/mood, pain, and fatigue (Fukuda et al, 1998; Steele, 2000; Gwini et al, 2016). Although GWI affects approximately $30 \%$ of U.S. military service members deployed to the Persian Gulf theater in 1990-1991, the underlying cause for these symptoms remains unclear (Steele et al, 2015).

Exposure to chemicals and toxins has been hypothesized as possible underlying causes or factors associated with GWI (Steele, 2000). Many of the toxins (Fiedler et al, 1996) and events to which service members were exposed (e.g., organophosphates, chemical nerve agents, and blast) have been shown to be or are suspected to have vestibulotoxic effects (Hsu et al, 2015; Littlefield et al, 2016). In a previous research study, Roland et al (2000) examined dizziness symptoms and vestibular test results in a small cohort of Gulf War veterans. Their research demonstrated that a high proportion of individuals reported dizziness symptoms. Objective vestibular and balance test results were also suggestive of possible vestibular impairment; however, the findings are difficult to interpret because conventional vestibular measurement parameters (i.e., rotary chair gain and phase and caloric unilateral weakness) were not reported. Despite these limitations, their work provides preliminary evidence that vestibular dysfunction may be present in GWI and potentially may underlie some symptoms of GWI (Roland et al, 2000).

The investigation of dizziness/vertigo symptoms and vestibular dysfunction in Gulf War veterans is complicated by the presence of mental health comorbidities and traumatic brain injury (TBI). Gulf War veterans, and deployed veterans in general, experience higher rates of anxiety, posttraumatic stress disorder (PTSD), depression, and TBI (Black et al, 2004; Bierer et al, 2015; McKenzie et al, 2015; Yee et al, 2017) when compared with civilian and nondeployed military populations.

The coexistence of symptoms of dizziness and symptoms associated with mental health conditions often makes it difficult to establish the independent contributions of underlying conditions. The comor- bidity of symptoms of dizziness (and/or concurrent vestibular impairment) and mental health conditions such as depression and anxiety have been well documented (Weidt et al, 2014). Furthermore, symptoms associated with mental health conditions can impact a person's experience of concurrent symptoms of dizziness (Kahraman et al, 2017) and can also negatively impact recovery from symptoms of vertigo/dizziness (Herdman et al, 2012). Although dizziness and vertigo can be symptoms of peripheral vestibular dysfunction, these symptoms are not exclusive to vestibular pathology and may be associated with a variety of underlying problems (Dretsch et al, 2016). In addition, as veterans with GWI age, their unresolved symptoms potentially become further confounded by other disorders (e.g., diabetes) which may increase symptom burden, lower quality of life, and may further hamper our ability to tease apart the underlying pathophysiology of the symptoms (Gwini et al, 2016).

A recent series of studies has considered the impact of TBI on the presence of chronic multisymptom illness in Gulf War veterans, finding increased self-reported mild TBI is related to an increase in physical symptom burden (Yee et al, 2017). These authors suggested that the combined effects of head injury and exposures in Gulf War veterans may promote persistent neuroinflammation thought to be at the root of GWI symptoms (Yee et al, 2017). Mild TBI has also been shown to have a persistent negative influence on vertigo/dizziness symptoms in approximately one-third of individuals (Fife and Kalra, 2015).

The present study is a descriptive case series of Gulf War veterans clinically evaluated at a tertiary referral center. The study consisted of two parts: a retrospective chart review followed by a prospective telephone interview. The purpose of this study was to characterize the presence of dizziness and vertigo symptoms using validated self-report instruments while accounting for mental health comorbidities in our clinical population of Gulf War veterans.

\section{METHODS}

\section{Setting}

The War Related Illness and Injury Study Center (WRIISC) at the Veterans Affairs (VA) New Jersey Health Care System is a tertiary evaluation center that specializes in post-deployment health care. Veterans are 
referred by VA clinicians and are evaluated by the WRIISC's multidisciplinary team of practitioners for exposure concerns and chronic medically unexplained symptoms that may be related to deployment (Lincoln et al, 2006).

\section{Participants}

Participants represent a follow-up sample of a case series of Gulf War veterans who were clinically evaluated at the New Jersey WRIISC between 2011 and 2016 (Fox et al, 2017). Veterans were included in the initial case series (a chart review) if they were deployed to the first Gulf War (1990-1991) and if they were evaluated by the New Jersey WRIISC between 2010 and 2016. There were no exclusion criteria. All veterans identified and included in the initial case review $(\mathrm{n}=153)$ were contacted by letter and follow-up phone call and invited to participate in this follow-up arm of the study. Sixty-one (39.8\%) veterans agreed to participate and completed a 70-min interview over the phone using validated questionnaires. Participants were asked about current medications and whether they had obtained any new head injuries (see Questionnaires) since their visit to the WRIISC. All participants provided verbal consent before their inclusion in the study. The study was approved by the Institutional Review Board of the VA Medical Center.

For this report, we only included participants who met criteria for GWI according to the definition endorsed by the CDC (Fukuda et al, 1998). To meet the CDC criteria for GWI, participants reported moderate to severe symptoms in two of three symptom clusters (fatigue, pain, and cognitive/mood) from the Kansas questionnaire of GWI symptoms, symptom onset, and severity (Steele, 2000).

\section{Questionnaires}

Dizziness and vertigo symptoms were assessed using the Vertigo Symptom Scale (VSS). This instrument is commonly used to analyze patient reports of vertigo/ dizziness (Fong et al, 2015). Developed by Yardley et al (1992), it demonstrates high internal validity and test-retest reliability (Fong et al, 2015). The 15-item questionnaire yields three scores (range 0-60): a total score, a vertigo score, and an anxiety score. Higher scores indicate greater symptoms of dizziness. A score of 12 (Yardley et al, 1992) on the total score scale is considered suggestive of severe symptoms of dizziness. The vertigo scale has been validated by several researchers in different populations with vestibular dysfunction (Fong et al, 2015) and the anxiety scale has likewise been validated multiple times in different populations with anxiety (Yardley et al, 1992; Radziej et al, 2015).

Symptoms of depression were measured using the Patient Health Questionnaire depression scale (PHQ-8). This eight-item questionnaire is used as a rapid and effec- tive tool for screening for major depressive disorders (Dhingra et al, 2011). The questionnaire is scored from 0 to 24 , with higher scores indicating more symptoms of depression; a score of 10 or more is considered suggestive of major depression (Dhingra et al, 2011). For our study, we used the suggested threshold of 10 or more as well as endorsement of anhedonia (score of 2 or more on question 1 or 2 ) to be categorized as having depression (Dhingra et al, 2011).

Symptoms of PTSD were measured using the PTSD Checklist (PCL-5) for Diagnostic and Statistical Manual of Mental Disorders, Fifth Edition (DSM-5). This version of the PCL reflects the diagnostic criteria established by DSM-5 and is a validated instrument designed to identify PTSD within a military population. There are 20 items, and higher scores (range 0-80) indicate greater symptom severity. For this study, PTSD was identified using a cutoff score of 33 or more, combined with the presentation of specific symptom clusters as defined by DSM-5 (Blevins et al, 2015).

Physical symptom burden was measured using the Patient Health Questionnaire somatization scale (PHQ15). This 15 -item questionnaire is designed to evaluate the severity of somatic symptoms (Kroenke et al, 2002). Scores range from 0 to 30 , and higher scores reflect greater overall physical symptom burden (McAndrew et al, 2017).

Symptoms of anxiety were measured using the Beck Anxiety Inventory (BAI), a 21-item questionnaire designed to identify anxiety (Beck and Steer, 1993). Scored on a scale of $0-63$, anxiety is categorized as minimal to severe with higher scores, indicating more symptoms of anxiety. Participants were categorized as having anxiety symptoms if they had a score of 16 or higher, consistent with a rating of "moderate anxiety" as defined by the authors of the questionnaire (Beck and Steer, 1993).

Traumatic brain injury was identified during the chart review using the Defense and Veterans Brain Injury Center (DVBIC) screening tool (Schwab et al, 2007). This three-item screening tool is widely used throughout the VA to screen for TBI. A positive response to any item in question 1 (injury) and a positive response to any item in question 2 (concussive symptoms at the time of injury) results in a positive screen (Schwab et al, 2007). At the time of the phone interview, the participants were asked whether they had experienced any new head injuries since their visit at the WRIISC. If they replied positively, they were then screened for TBI resulting from the recent head injuries using question 2 of the DVBIC screening tool.

\section{Analyses}

We used descriptive statistics (mean and standard deviation, frequency/percentage distributions) to describe our sample. All analyses were conducted using SPSS (SPSS Inc. Released 2016. IBM SPSS Statistics for Windows, Version 24.0; IBM Corp., Armonk, NY). 


\section{RESULTS}

$\mathrm{F}$ ifty veterans met the CDC criteria for GWI and were included in the analysis. The majority were male $(\mathrm{n}=46,92 \%)$, were on average $49( \pm 6.4)$ years old, and were taking $8.9( \pm 6.8)$ medications. The most commonly reported medications were nutritional supplements $(\mathrm{n}=33,54 \%)$, followed by antidepressants $(\mathrm{n}=$ $29,48 \%)$, cardiovascular medications $(\mathrm{n}=28,46 \%)$, and allergy medications $(\mathrm{n}=21,41 \%)$. Almost one quarter of the participants $(\mathrm{n}=12,24 \%)$ reported taking greater than ten medications (excluding supplements). Forty-two percent $(n=21)$ met the DVBIC screening criteria for TBI. One veteran reported a new head injury since WRIISC visit, but his lifetime TBI status remained unchanged. For our sample, TBI screening status did not further differentiate participants from other subgroups they belonged to and, therefore, this variable was not included in the descriptive analyses.

Table 1 provides a frequency distribution of variables for the total group, separated by mental health symptoms. Thirty-two percent $(\mathrm{n}=16)$ of our sample met the screening criteria for PTSD, $42 \%(n=24)$ of our sample met the screening criteria for depression, and $92 \%$ $(n=46)$ of our sample met the screening criteria for anxiety. Overall, $90 \%(n=45)$ of our participants reported symptoms of dizziness, which met the VSS screening threshold $(>12)$ for "severe dizziness." The mean total score for the VSS was $25( \pm 11.3)$ in the overall sample and the mean total score for the PHQ-15 was 18.1 ( \pm 4.3 ).

Table 2 presents average responses to the individual items of the VSS for the full sample, the depression subgroups, and the PTSD subgroups. The anxiety subgroup is not represented, given the small sample size of participants included in the groups (four screening negative compared with 46 screening positive). The individual VSS questions are broken into their respective subscales (vertigo or anxiety) as defined by Yardley et al (1992). In addition, we present mean differences in individual item scores between those screening positive or negative for depression or PTSD. The most commonly endorsed symptom on the VSS was question 7 (headache or pressure in the head), which was on average reported as 2.7 out of 4 . Items that were strongly endorsed (e.g., 2 or more out of 4 ) on the vertigo subscale included questions 1 (spinning $>20 \mathrm{~min}$ ), 8 (veering or staggering), 13 (unsteady $>20$ $\min$ ), and 15 (swimmy $>20 \mathrm{~min}$ ). Items that were strongly endorsed on the anxiety subscale included questions 2 (hot or cold spells) and 11 (excessive sweating).

\section{DISCUSSION}

$\mathrm{O}$ ur findings demonstrate high scores on the VSS in our sample of veterans with GWI. Mean total VSS scores in our sample were $24.5( \pm 11.8)$ and $90 \%$ of our sample exceeded the threshold $(>12)$ for detecting "severe dizziness" on the VSS. The most commonly reported symptom was headache or pressure in the head; however, our participants frequently reported symptoms consistent with dizziness and vertigo (e.g., veering or staggering, unsteady/spinning/swimmy $>20 \mathrm{~min}$ ). Furthermore, the symptoms most commonly reported on the anxiety subscale (hot or cold spells, excessive sweating, and pressure in the head) are more related to physical symptoms associated with anxiety than to cognitive symptoms associated with anxiety.

These findings are consistent with the previous report by Roland et al (2000), although the self-reported symptoms in our study exceed those reported on average by Roland et al (2000). There are many possible reasons for these differences; in addition to the difference between our populations (theirs including controlled scientific groups, ours representing a clinical cohort), our study differed from theirs in the way that GWI was categorized, the questions used to evaluate symptoms, and the amount of time the veteran had been experiencing their symptoms (by roughly ten years). Although vertigo and dizziness are not exclusively symptoms of vestibular dysfunction, Gulf War veterans were exposed to many different potentially ototoxic incidents (Fiedler et al, 1996), and the possible contribution of vestibular dysfunction to GWI should be further explored in future research.

Our sample comprised veterans who were evaluated at a tertiary medical clinic for chronic post-deployment health concerns, all of whom met the criteria for GWI. Nearly one-quarter of them reported taking ten or more medications (although the mean number of medications was 8.9 ) and $\sim 40 \%$ of them met the DVBIC screening criteria for TBI. The mean PHQ-15 score in our sample was $18.1( \pm 4.3)$, which greatly exceeds scores in the general population $(4.6 \pm 3.6$ for men [Hinz et al, 2017]). We interpret the high PHQ-15 scores to reflect a substantial burden of somatic symptoms in this population, consistent with their diagnosis of GWI, and support the internal validity of our findings.

We sought to characterize the presence of vertigo and dizziness symptoms of veterans with GWI while accounting for mental health comorbidities. Within our clinical sample, we saw that approximately one-third of our participants met the screening criteria for PTSD and nearly half of our participants met the screening criteria for depression. This is consistent with previous reports of veterans evaluated at the WRIISC (Lincoln et al, 2006). We also saw that approximately $90 \%$ of our sample met the screening criteria for anxiety. This prevalence rate is higher than has been previously reported (Storzbach et al, 2000); however, we believe this is because of limitations with the use of the BAI in this population. Previous authors have documented that the BAI total score may not accurately capture the cognitive aspects of anxiety in individuals with increased somatic 


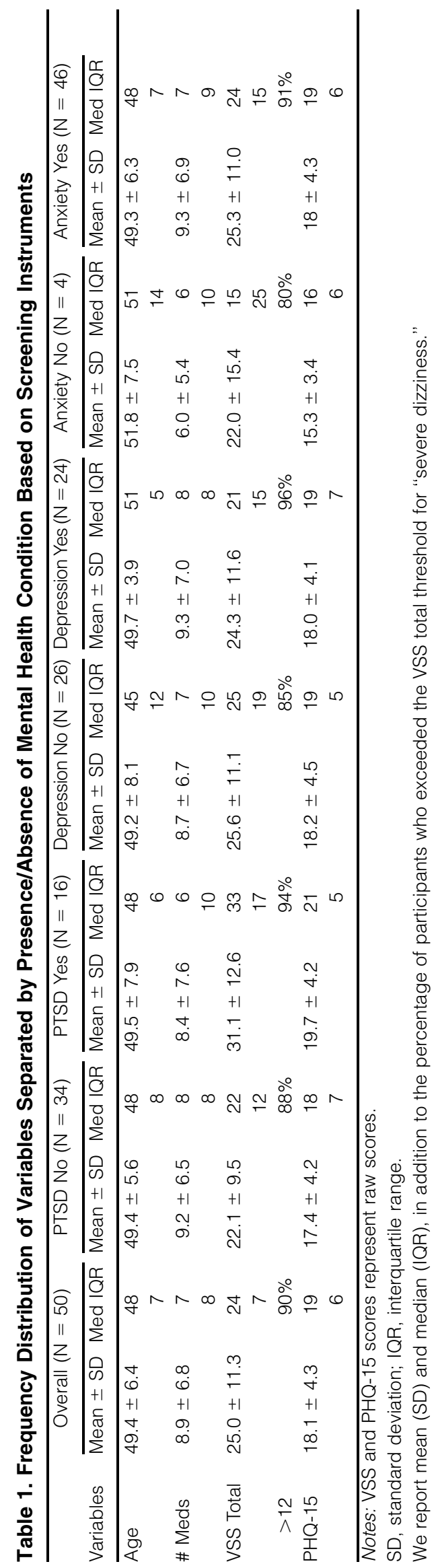


Table 2. Mean Responses to Individual Items on VSS, Separated by Presence/Absence of Mental Health Condition Based on Screening Instruments

\begin{tabular}{|c|c|c|c|c|c|c|c|c|}
\hline Question Number & VSS Question & $\begin{array}{l}\text { Overall } \\
(\mathrm{N}=50)\end{array}$ & $\begin{array}{l}\text { Depression } \\
\text { No }(\mathrm{N}=26)\end{array}$ & $\begin{array}{c}\text { Depression } \\
\text { Yes }(\mathrm{N}=24)\end{array}$ & $\begin{array}{c}\text { Mean } \\
\text { Difference } \\
(\mathrm{Y} / \mathrm{N})\end{array}$ & $\begin{array}{l}\text { PTSD No } \\
(\mathrm{N}=34)\end{array}$ & $\begin{array}{l}\text { PTSD Yes } \\
(N=16)\end{array}$ & $\begin{array}{c}\text { Mean } \\
\text { Difference } \\
(\mathrm{Y} / \mathrm{N})\end{array}$ \\
\hline \multicolumn{9}{|l|}{ Vertigo subscale } \\
\hline 1 & Spinning $<20 \mathrm{~min}$ & $1.9 \pm 1.3$ & $1.8 \pm 1.4$ & $1.9 \pm 1.2$ & 0.1 & $1.7 \pm 1.2$ & $2.3 \pm 1.4$ & 0.6 \\
\hline 3 & Nausea, vomiting & $1.7 \pm 1.5$ & $1.7 \pm 1.6$ & $1.8 \pm 1.5$ & 0.1 & $1.5 \pm 1.4$ & $2.1 \pm 1.6$ & 0.6 \\
\hline 4 & Spinning $>20 \mathrm{~min}$ & $0.6 \pm 0.8$ & $0.5 \pm 0.8$ & $0.6 \pm 0.9$ & 0.1 & $0.4 \pm 0.7$ & $0.8 \pm 1.0$ & 0.4 \\
\hline 6 & Swimmy all day & $0.7 \pm 1.2$ & $0.8 \pm 1.3$ & $0.5 \pm 0.9$ & 0.3 & $0.4 \pm 0.7$ & $1.3 \pm 1.6$ & 0.9 \\
\hline 8 & Veering or staggering & $2.4 \pm 1.5$ & $2.5 \pm 1.6$ & $2.4 \pm 1.4$ & 0.1 & $2.5 \pm 1.4$ & $2.3 \pm 1.7$ & 0.2 \\
\hline 10 & Unsteady $>20 \mathrm{~min}$ & $1.2 \pm 1.5$ & $1.5 \pm 1.6$ & $0.9 \pm 1.5$ & 0.6 & $1.0 \pm 1.5$ & $1.6 \pm 1.7$ & 0.6 \\
\hline 13 & Unsteady $<20$ min & $2.2 \pm 1.4$ & $2.1 \pm 1.6$ & $2.4 \pm 1.3$ & 0.3 & $2.0 \pm 1.4$ & $2.8 \pm 1.4$ & 0.8 \\
\hline 15 & Swimmy $<20$ min & $1.9 \pm 1.3$ & $1.8 \pm 1.4$ & $2.0 \pm 1.3$ & 0.2 & $1.7 \pm 1.2$ & $2.3 \pm 1.4$ & 0.6 \\
\hline \multicolumn{9}{|l|}{ Anxiety subscale } \\
\hline 2 & Hot or cold spells & $2.3 \pm 1.5$ & $2.5 \pm 1.4$ & $2.2 \pm 1.6$ & 0.3 & $2.2 \pm 1.5$ & $2.8 \pm 1.5$ & 0.6 \\
\hline 5 & $\begin{array}{l}\text { Heart pounding or } \\
\text { fluttering }\end{array}$ & $1.6 \pm 1.3$ & $1.8 \pm 1.3$ & $1.4 \pm 1.3$ & 0.4 & $1.3 \pm 1.2$ & $2.2 \pm 1.3$ & 0.9 \\
\hline 7 & $\begin{array}{l}\text { Headache or pressure } \\
\text { in the head }\end{array}$ & $2.7 \pm 1.4$ & $2.5 \pm 1.3$ & $2.9 \pm 1.4$ & 0.4 & $2.5 \pm 1.4$ & $3.2 \pm 1.1$ & 0.7 \\
\hline 9 & Difficulty breathing & $1.7 \pm 1.4$ & $1.9 \pm 1.6$ & $1.5 \pm 1.3$ & 0.4 & $1.5 \pm 1.4$ & $2.2 \pm 1.5$ & 0.7 \\
\hline 11 & Excessive sweating & $2.0 \pm 1.6$ & $2.3 \pm 1.6$ & $1.7 \pm 1.6$ & 0.6 & $2.0 \pm 1.6$ & $2.0 \pm 1.6$ & 0.0 \\
\hline 12 & Feeling faint & $0.9 \pm 1.1$ & $0.7 \pm 1.0$ & $1.1 \pm 1.3$ & 0.4 & $0.7 \pm 1.0$ & $1.3 \pm 1.4$ & 0.6 \\
\hline 14 & Pain in heart or chest & $1.2 \pm 1.2$ & $1.3 \pm 1.4$ & $1.0 \pm 1.0$ & 0.3 & $0.7 \pm 0.8$ & $2.0 \pm 1.4$ & 1.3 \\
\hline
\end{tabular}

complaints but may instead capture somatic symptoms associated with, but not intrinsic to, anxiety alone (Julian, 2011). Examining the individual items on the BAI compared with a more extensive list of GWI symptoms commonly reported by veterans (Steele, 2000; Gwini et al, 2016), we see a strong overlap (see Table 3), supporting this explanation. Therefore, although we report the findings, we conclude that the BAI is not a good measure in veterans with GWI and possibly other groups with significant somatic complaints (e.g., chronic fatigue syndrome and fibromyalgia).

The relationships between depression, anxiety, PTSD, and symptoms of dizziness and vertigo warrant some discussion. Depression has been reported to be elevated within a veteran population (Lincoln et al, 2006) and is four times more likely to be present in individuals with

\section{Table 3. Individual Items on GWI Questionnaire and Beck Anxiety Inventory}

\begin{tabular}{ll}
\hline GWI Questionnaire & \multicolumn{1}{c}{ Beck Anxiety Inventory } \\
\hline Fatigue & Numbness or tingling \\
Feeling unwell after physical exercise or exertion & Wobbliness in legs \\
Problems getting to sleep or staying asleep/not feeling rested after sleeping & Dizzy or lightheaded \\
Pain in the joints/stiffness in the joints/pain in the muscles/body pain & Unsteady \\
Headaches & Hands trembling \\
Feeling dizzy, lightheaded, or faint & Shaky \\
Eyes sensitive to light/blurred or double vision & Faint \\
Numbness or tingling in the extremities/tremors or shaking & Feeling hot \\
Low tolerance for heat/cold & Indigestion or discomfort in the abdomen \\
Night sweats & Face flushed \\
Physical or mental symptoms after breathing in certain smells/chemicals & Sweating not due to heat \\
Skin rashes & Heart pounding or racing \\
Diarrhea & Feelings of choking \\
Nausea or upset stomach/abdominal pain or cramping & Difficulty breathing \\
Difficulty breathing or catching your breath & Unable to relax \\
Frequent coughing when not having a cold/wheezing in the chest & Fear of the worst happening \\
Trouble finding words when speaking & Terrified \\
Difficulty remembering recent information & Nervous \\
Difficulty concentrating & Fear of losing control \\
Feeling down or depressed/feeling moody/feeling anxious & Scared \\
Feeling irritable or having angry outbursts & Fear of dying \\
\hline
\end{tabular}

Notes: Items that are similar across the questionnaires are in bold. 
multisymptom illness or PTSD (McKenzie et al, 2015), negatively impacting quality of life.

The relationship between vestibular symptoms and anxiety is well established (Herdman et al, 2012). Indeed, there are specific underlying anatomical structures that mediate communication between the vestibular system and anxiety pathways (e.g., the parabrachial nucleus and sympathetic nervous system activation) (Balaban, 2002; Radziej et al, 2015). The presence of anxiety can exacerbate an individual's experience of symptoms of vertigo and dizziness and can negatively impact the rehabilitation and recovery from symptoms of vertigo and dizziness (Herdman et al, 2012).

PTSD is categorized as an anxiety disorder, but it has been suggested that there are relationships between PTSD and symptoms of vertigo and dizziness that extend beyond the relationship suggested by an anxiety disorder. Recent studies have contextualized disruptions within central vestibular pathways for individuals with PTSD (Harricharan et al, 2017; Tigno et al, 2017). There are several possible reasons for an increased experience of vertigo symptoms in veterans with PTSD. Physical symptoms have generally been reported to be worse in individuals with PTSD than in individuals without PTSD (Pacella et al, 2013). This poorer overall health and functioning of individuals with PTSD is attributed to mechanisms associated with chronic stress, including disrupted sleep (Pacella et al, 2013), and may result in greater vertigo symptoms, as well. Another explanation is found in recent studies identifying differences in central vestibular pathways in civilians with PTSD (Harricharan et al, 2017) and in veterans with PTSD and comorbid TBI (Tigno et al, 2017). These studies suggest that different injuries (blast, vascular injury due to sudden hypertension) may disrupt central vestibular pathways in similar ways, resulting in both vestibular dysfunction and PTSD. Our findings, in this context, further support the need for thorough investigation of the relationships among vertigo symptoms, vestibular function, and PTSD.

We acknowledge several limitations, including the crosssectional nature of our study and lack of a comparison group of participants without GWI. All the participants included in our study were initially evaluated at a tertiary facility specializing in medically unexplained symptoms, and our findings are, therefore, not representative of the larger Gulf War veteran cohort. In addition, our sample may be biased by self-selection of those veterans willing to participate in a 70-min phone interview. Furthermore, to our knowledge, none of these questionnaires has been validated for verbal administration and this could have impacted our findings. Finally, we examined self-reported data and although we described the medication usage in our sample, we were unable to systematically rule out the possible influence of medications on symptoms of vertigo/dizziness. However, we believe that despite the limitations, the descriptive nature of this study combined with the use of established, commonly used instruments to capture self-report, and the relatively large clinical sample of this rare condition lend relevance and importance to our study.

In conclusion, this study corroborates earlier findings of a high self-reporting of symptoms of dizziness in veterans with GWI. It will be important to follow up with rigorous research using objective measures of vestibular function to further characterize the prevalence and impact of vestibular dysfunction in the population of veterans with GWI. Symptoms of dizziness do not inherently mean that there is underlying vestibular dysfunction, but if these symptoms are present in clinical evaluation for GWI, vestibular testing appears to be justified. Second, our findings suggest that the use of BAI as a measure of anxiety in GWI may be problematic, similar to using BAI in other groups with chronic conditions. Clinicians and researchers should consider additional or alternative measures of anxiety in populations with high physical symptom burden. Finally, we highlight the relationships among depression, anxiety, PTSD, and symptoms of vertigo and dizziness in veterans with GWI, which warrant further examination using objective testing.

\section{REFERENCES}

Balaban CD. (2002) Neural substrates linking balance control and anxiety. Physiol Behav 77:469-475.

Beck AT, Steer RA. (1993) Beck Anxiety Inventory Manual. San Antonio, TX: Psychological Corporation.

Bierer LM, Ivanov I, Carpenter DM, Wong EW, Golier JA, Tang CY, Yehuda R. (2015) White matter abnormalities in Gulf War veterans with posttraumatic stress disorder: a pilot study. Psychoneuroendocrinology 51:567-576.

Black DW, Carney CP, Peloso PM, Woolson RF, Schwartz DA, Voelker MD, Barrett DH, Doebbeling BN. (2004) Gulf War veterans with anxiety: prevalence, comorbidity, and risk factors. $E p$ idemiology 15:135-142.

Blevins CA, Weathers FW, Davis MT, Witte TK, Domino JL. (2015) The posttraumatic stress disorder checklist for DSM-5 (PCL-5): development and initial psychometric evaluation. $J$ Trauma Stress 28:489-498.

Dhingra SS, Kroenke K, Zack MM, Strine TW, Balluz LS. (2011) PHQ-8 days: a measurement option for DSM-5 major depressive disorder (MDD) severity. Popul Health Metr 9:11.

Dretsch MN, Williams K, Emmerich T, Crynen G, Ait-Ghezala G, Chaytow H, Mathura V, Crawford FC, Iverson GL. (2016) Brainderived neurotropic factor polymorphisms, traumatic stress, mild traumatic brain injury, and combat exposure contribute to postdeployment traumatic stress. Brain Behav 6:e00392.

Fiedler N, Kipen H, Natelson B, Ottenweller J. (1996) Chemical sensitivities and the Gulf War: Department of Veterans Affairs Research Center in basic and clinical science studies of environmental hazards. Regul Toxicol Pharmacol 24:S129-S138. 
Fife TD, Kalra D. (2015) Persistent vertigo and dizziness after mild traumatic brain injury. Ann N Y Acad Sci 1343:97-105.

Fong E, Li C, Aslakson R, Agrawal Y. (2015) Systematic review of patient-reported outcome measures in clinical vestibular research. Arch Phys Med Rehabil 96:357-365.

Fox A, Helmer D, Tseng C, Patrick-DeLuca L, Osinubi OY. (2017) Report of autonomic symptoms in a clinical sample of veterans with Gulf War illness. Mil Med 183:e179-e785.

Fukuda K, Nisenbaum R, Stewart G, Thompson WW, Robin L, Washko RM, Noah DL, Barrett DH, Randall B, Herwaldt BL, Mawle AC, Reeves WC. (1998) Chronic multisymptom illness affecting air force veterans of the Gulf War. J Am Med Assoc 280: 981-988.

Gwini SM, Forbes AB, Sim MR, Kelsall HL. (2016) Multisymptom illness in Gulf War veterans: a systematic review and metaanalysis. J Occup Environ Med 58:659-667.

Harricharan S, Nicholson AA, Densmore M, Theberge J, McKinnon MC, Neufeld RWJ, Lanius RA. (2017) Sensory overload and imbalance: resting-state vestibular connectivity in PTSD and its dissociative subtype. Neuropsychologia 106:169-178.

Herdman SJ, Hall CD, Delaune W. (2012) Variables associated with outcome in patients with unilateral vestibular hypofunction. Neurorehabil Neural Repair 26:151-62.

Hinz A, Ernst J, Glaesmer H, Brahler E, Rauscher FG, Petrowski K, Kocalevent RD. (2017) Frequency of somatic symptoms in the general population: normative values for the patient health questionnaire-15 (PHQ-15). J Psychosom Res 96:27-31.

Hsu PC, Cheng PW, Young YH. (2015) Ototoxicity from organic solvents assessed by an inner ear test battery. J Vestib Res 25: 177-183.

Julian LJ. (2011) Measures of anxiety: state-trait anxiety inventory (STAI), beck anxiety inventory (BAI), and hospital anxiety and depression scale-anxiety (HADS-A). Arthritis Care Res 63(11), (Suppl):S467-S472.

Kahraman SS, Arli C, Copoglu US, Kokacya MH, Colak S. (2017) The evaluation of anxiety and panic agarophobia scores in patients with benign paroxysmal positional vertigo on initial presentation and at the follow-up visit. Acta Otolaryngol 137:485-489.

Kroenke K, Spitzer RL, Williams JB. (2002) The PHQ-15: validity of a new measure for evaluating the severity of somatic symptoms. Psychosom Med 64:258-266.

Lincoln A, Helmer DA, Schneiderman A, Li M, Copeland HL, Prisco M, Wallin M, Kang HK, Natelson B. (2006) The war-related illness and injury study centers: a resource for deployment-related health concerns. Mil Med 171:577.

Littlefield PD, Pinto RL, Burrows HL, Brungart DS. (2016) The vestibular effects of repeated low-level blasts. J Neurotrauma 33:71-81.

McAndrew LM, Phillips LA, Helmer DA, Maestro K, Engel CC, Greenberg LM, Anastasides N, Quigley KS. (2017) High healthcare utilization near the onset of medically unexplained symptoms. $J$ Psychosom Res 98:98-105.

McKenzie DP, Sim MR, Clarke DM, Forbes AB, Ikin JF, Kelsall HL. (2015) Developing a brief depression screen and identifying associations with comorbid physical and psychological illness in Australian Gulf War veterans. J Psychosom Res 79: $566-573$.

Pacella ML, Hruska B, Delahanty DL. (2013) The physical health consequences of PTSD and PTSD symptoms: a meta-analytic review. J Anxiety Disord 27:33-46.

Radziej K, Schmid G, Dinkel A, Zwergal A, Lahmann C. (2015) Psychological traumatization and adverse life events in patients with organic and functional vestibular symptoms. J Psychosom Res 79:123-129.

Roland PS, Haley RW, Yellin W, Owens K, Shoup AG. (2000) Vestibular dysfunction in Gulf War syndrome. Otolaryngol Head Neck Surg 122:319-329.

Schwab KA, Ivins B, Cramer G, Johnson W, Sluss-Tiller M, Kiley K, Lux W, Warden D. (2007) Screening for traumatic brain injury in troops returning from deployment in Afghanistan and Iraq: initial investigation of the usefulness of a short screening tool for traumatic brain injury. J Head Trauma Rehabil 22: 377-389.

Steele L. (2000) Prevalence and patterns of Gulf War illness in Kansas veterans: association of symptoms with characteristics of person, place, and time of military service. Am J Epidemiol 152:992-1002.

Steele L, Lockridge O, Gerkovich MM, Cook MR, Sastre A. (2015) Butyrylcholinesterase genotype and enzyme activity in relation to Gulf War illness: preliminary evidence of gene-exposure interaction from a case-control study of 1991 Gulf War veterans. Environ Health 14:4.

Storzbach D, Campbell KA, Binder LM, McCauley L, Anger WK, Rohlman DS, Kovera CA. (2000) Psychological differences between veterans with and without Gulf War unexplained symptoms. Portland environmental hazards research center. Psychosom Med 62: 726-735.

Tigno Jr. TA, Armonda RA, Bell RS, Severson 3rd MA . (2017) The vestibulocochlear bases for wartime posttraumatic stress disorder manifestations. Med Hypotheses 106:44-56.

Weidt S, Bruehl AB, Straumann D, Hegemann SC, Krautstrunk G, Rufer M. (2014) Health-related quality of life and emotional distress in patients with dizziness: a cross- sectional approach to disentangle their relationship. BMC Health Serv Res 14:317.

Yardley L, Masson E, Verschuur C, Haacke N, Luxon L. (1992) Symptoms, anxiety and handicap in dizzy patients: development of the vertigo symptom scale. J Psychosom Res 36:731-741.

Yee MK, Janulewicz PA, Seichepine DR, Sullivan KA, Proctor SP, Krengel MH. (2017) Multiple mild traumatic brain injuries are associated with increased rates of health symptoms and Gulf War illness in a cohort of 1990-1991 Gulf War veterans. Brain Sci 7:E79. 